\title{
Water treadmill training attenuates blood-spinal cord barrier disruption by promoting angiogenesis and inhibiting MMP-2/9 expression following spinal cord injury
}

\section{Xinwang Ying}

The Second Affiliated Hospital and Yuying Children'Hospital of Wenzhou Medical University

\section{Qingfeng Xie}

The Second Affiliated Hospital and Yuying Children'Hospital of Wenzhou Medical University

\section{Shengcun Li}

The Second Affiliated Hospital and Yuying Children' Hospital of Wenzhou Medical University

\section{Xiaolan Yu}

The Second Affiliated Hospital and Yuying Children's Hospital of Wenzhou Medical University

\section{Kecheng Zhou}

The Second Affiliated Hospital and Yuying Children's Hospital of Wenzhou Medical University Jingjing Yue

The Second Affiliated Hospital and Yuying Children's Hospital of Wenzhou Medical University

\section{Xiaolong Chen}

The Second Affiliated Hospital and Yuying Children's Hospital of Wenzhou Medical University

\section{Wenzhan Tu}

The Second Affiliated Hospital and Yuying Children's Hospital of Wenzhou Medical University

\section{Guanhu Yang}

The Second Affiliated Hospital and Yuying Children's Hospital of Wenzhou Medical University

\section{Songhe Jiang ( $\nabla_{\text {jiangsonghe@wmu.edu.cn ) }}$}

The Second Affiliated Hospital and Yuying Children's Hospital of Wenzhou Medical University

\section{Research}

Keywords: Spinal cord injury, BSCB, Underwater-treadmill training, Vascular regeneration, MMP-2/9

Posted Date: September 11th, 2020

DOl: https://doi.org/10.21203/rs.3.rs-37000/v2

License: (c) (i) This work is licensed under a Creative Commons Attribution 4.0 International License. Read Full License 
Version of Record: A version of this preprint was published at Fluids and Barriers of the CNS on November 25th, 2020. See the published version at https://doi.org/10.1186/s12987-020-00232-1. 


\section{Abstract}

Background: The permeability of blood-spinal cord barrier (BSCB) is mainly determined by the junction complex of adjacent endothelial cells, including tight junction (TJ) and adhesion junction (AJ), which can be severely damaged after spinal cord injury $(\mathrm{SCl})$. Exercise training is a recognized method for the treatment of SCl. The destruction of the BSCB mediated by matrix metalloproteinase (MMP) leads to inflammation, neurotoxin production, and apoptosis of neurons. The failure of effective regeneration of new blood vessels is also an important reason for the difficulty of recovery after SCl. We introduced water treadmill training (TT) for the first time, which can help SCI rats successfully exercise, and we explored the role of TT in promoting the ability to exercise after $\mathrm{SCl}$ and its possible mechanism.

Methods: Sprague-Dawley $(200-250 \mathrm{~g})$ rats were randomly divided into three groups. SCI models were established and rats underwent TT after SCl. Animals were sacrificed $7 \mathrm{~d}$ or $14 \mathrm{~d}$ post-surgery. The degree of neurological deficit, water content, BSCB permeability, apoptosis, protein expression and ultrastructure of vascular endothelial cells (EC) were assessed by the Basso-Beattie-Bresnahan (BBB) motor rating scale, haematoxylin-eosin (HE) staining, Evans blue (EB) staining, TUNEL staining, Western blot (WB) experiments, and immunofluorescence and transmission electron microscopy (TEM).

Results: Our experiments show that TT reduces the permeability of BSCB and decreased tissue structure damage. TT improved functional recovery and it has significant significance when compared with the M group after SCl; TJ and AJ proteins increased significantly after TT training in $\mathrm{SCl}$ rats. TT training reduced apoptosis induced by SCl. TT can promote angiogenesis and the expressions of MMP-2 and MMP-9 were significantly inhabited by TT after SCl.

Conclusions: In this study, the results indicate that TT promotes functional recovery partly for the following reasons: (1) TT protects residual BSCB structure from further damage; (2) it promotes vascular regeneration; and (3) it inhibits the expression of MMP-2/9 to mitigate BSCB damage.

\section{Background}

Spinal cord injury (SCl) places a heavy burden on society and the economy [1]. Currently, it can only be relieved by surgery, but it cannot be cured [2]. The basement membrane, pericytes and the terminal foot process of astrocytes constitute the barrier of the BSCB, which protects and regulates the parenchyma of the spinal cord $[3,4]$. After $\mathrm{SCl}$, the barrier is destroyed, which is accompanied by the infiltration of immune cells and neurotoxic products, resulting in the death of nerve cells, and permanent neurological dysfunction [5-7]. Therefore, it is crucial to identify an intervention that can effectively prevent the destruction of the BSCB after SCl.

MMP is a family of zinc-containing peptidases that degrade and reshape the extracellular matrix and other extracellular proteins, and it plays a key role in barrier function $[7,8]$. Studies have shown that MMP plays a key role in the destruction of the BBB/BSCB under pathological conditions, including SCI. Two important members of the MMP superfamily are MMP-2 and -9 [9]. MMP-9 can induce BSCB-related 
protein degradation, and upregulation of MMP-2 will lead to the initial opening of the BBB/BSCB $[10,11]$, while blocking MMP-9 activity will protect vascular permeability [12]. The expression of MMP-2/9 was still detected $7 \mathrm{~d}$ after SCI [13]. Vascular endothelial growth factor (VEGF) is a highly specific vascular endothelial cell growth factor that promotes vascular permeability, proliferation, and angiogenesis [14] It is a necessary angiogenic factor for embryonic development and neovascularization under many pathological conditions [15]. Several molecules have been proved to be angiogenic in vivo, but only VEGF is considered to be a secretory mitogen specific to vascular EC. At present, VEGF is the most eye-catching target for the study of vascular growth dynamics $[16,17]$.

Previous studies have mainly focused on drug treatment after SCI[18, 19]. However, this often has certain side effects, affecting the quality of life of patients. Therefore, it is very important for patients with $\mathrm{SCl}$ to find a safe, effective and healthy treatment. Exercise training is a non-traumatic rehabilitation method after SCl that can promote the functional recovery of paralyzed muscles [20-23]. Also, most studies have focused on the effect of drug therapy on the neurovascular system after $\mathrm{SCl}[24,25]$, ignoring the protection and functional recovery of the vascular system by exercise training. Based on this fact, starting from the actual clinical practice and simulating the exercise rehabilitation of clinical patients, our experimental team designed and invented a water treadmill for the first time which is suitable for rats to exercise after $\mathrm{SCl}$. Compared with a single swimming exercise, treadmill training can make rats passively and forcibly exercise at the initial stage, and can actively follow the treadmill in the middle and later stages of training. Due to the characteristics of high resistance, the ordinary treadmill training makes it difficult for rats to carry out rehabilitation exercise on it. And our water treadmill equipment can be a good combination of swimming and treadmill training, so as to make up for the above shortcomings. However, the protective effect of TT on SCl has not been reported in the literature; its effects on the BSCB are not clear.

\section{Method}

\section{Antibodies}

Anti-MMP-9, anti-Tubulin were purchased from Proteintech (Rosemont, IL, USA). Anti-VEGF and $\beta$-Actin were purchased from Abcam (330 Cambridge Science Park, Cambridge, UK). Anti-p120-Catenin, anti- $\beta$ Catenin, anti-ZO-1, anti-Occludin, and anti-Claudin-5 were purchased from Affinity (OH, USA).

\section{Animals}

A total of 123 adult male Sprague-Dawley rats $(200-250 \mathrm{~g})$ were purchased from Shanghai Laboratory Animal Center. The protocols were approved by the Animal Research Committee of Wenzhou Medical University. All animals were housed in a controlled environment and regularly fed with food and water. Rats were randomly divided into the following three groups: Sham $(n=41$; group $S) ; S C l(n=41$; group M); $\mathrm{SCl}+\mathrm{TT}(\mathrm{n}=41$; group TM$)$.

\section{SCI Model}


Rats were anaesthetized with $2 \%$ pentobarbital sodium $(30 \mathrm{mg} / \mathrm{kg})$, then were shaved and a $2 \mathrm{~cm}$ incision was made to expose the T10 segment of the spinal cord [26]. The exposed site was impacted with a New York University (NYU) Impactor $(10 \mathrm{~g} \times 20 \mathrm{~cm})$ in all groups except group S. The lower limb trembling contractions and tail wagging showed that $\mathrm{SCl}$ modeling was successful. Finally, the wound was sutured, disinfected with lodophor, and the bladder was emptied every morning and evening.

\section{Water treadmill training}

Our research group provided the initial design of a water treadmill (Wenzhou Xinglong Stainless Steel Co., LTD, Zhejiang, China) and submitted it for a patent. Rats were given adaptive training for three days before SCl. The depth of the water was adjusted to the xiphoid process of the sternum of the rat, the water temperature was set at $30^{\circ} \mathrm{C}$, and the speed of the water treadmill was maintained at 10-15 meters per minute. One day after $\mathrm{SCl}$, rats in the water treadmill training group began training, which lasted for 7 $\mathrm{d}$ or $14 \mathrm{~d}$ ( $5 \mathrm{~min} /$ round, 3 rounds in total, $5 \mathrm{~min}$ interval between rounds).

\section{Behavioral tests}

Two independent examiners who were blinded to the treatment groups conducted BBB scores on rats in an open field test. To put it simply, the BBB score has a total of 21 points, and the higher the score is, the closer the animal is to normal.

\section{Evaluation of BSCB permeability}

\section{Water content}

At $7 \mathrm{~d}$ and $14 \mathrm{~d}$ after $\mathrm{SCl}, 2 \%$ sodium pentobarbital was intraperitoneally injected to anaesthetise the animals $(n=5)$, and $0.5 \mathrm{~cm}$ of the T10 spinal cord was taken after perfusion with $0.9 \%$ normal saline. The degree of edema in this segment was measured by the dry and wet weight method as previously reported $[28,29]$.

\section{Evans blue dye assays}

According to previously reported methods [4, 8], rats $(n=5)$ were injected with EB dye ( $4 \mathrm{ml} / \mathrm{kg})$ by tail vein at $7 \mathrm{~d}$ and $14 \mathrm{~d}$ after $\mathrm{SCl}$, which was followed by $2 \%$ sodium pentobarbital anesthesia 2 hours late and $0.9 \%$ saline perfusion. Tissue containing $\mathrm{T} 10$ was soaked in $\mathrm{N}, \mathrm{N}^{\prime}$-dimethylformamide at $50^{\circ} \mathrm{C}$ for 72 hours. The concentration of dyes in the samples were determined based on a standard curve $(\mu \mathrm{g} / \mathrm{g})$. Tissues were cut into $15 \mu \mathrm{m}$ thick sections with a frozen microtome at $-20^{\circ} \mathrm{C}$, and then the sections were analysed. Quantitative analysis of data was performed with ImageJ software.

\section{Haematoxylin-eosin (HE)}

Briefly, tissues were removed from rats at 7 and $14 \mathrm{~d}$ after SCl, and then they were stored in $4 \%$ paraformaldehyde for 24 hours $\left(4^{\circ} \mathrm{C}\right)$. The spinal cord was immersed in a $0.1 \mathrm{~m}$ phosphate buffer 
solution and a $30 \%$ sucrose solution overnight $\left(4^{\circ} \mathrm{C}\right)$. Successive sections $(15 \mu \mathrm{m}$ thick) were frozen and stored for subsequent use. HE staining experiments were carried out with the appropriate kits.

\section{Western Blot Analysis}

Tissues containing T10 segments were put into a collection tube containing a mixture of PMSF and RIPA (100:1) and then were microfuged at $12,000 \mathrm{rpm}$ for $5 \mathrm{~min}$ at $4^{\circ} \mathrm{C}$. We extracted the supernatant and calculated the protein concentration with a BCA kit. The mixed solution was heated to $100^{\circ} \mathrm{C}$ for $10 \mathrm{~min}$. After electrophoretic transfer to membranes, primary and secondary antibodies were incubated successively. Then, the signal was digitally quantified.

\section{Immunofluorescence Staining}

After drying sections, they were washed 3 times for 15 minutes. After being treated with nonimmune goat serum for 1 hour, sections were incubated first with primary antibodies against rabbit anti-occludin antibody (1:100, Affinity, US), rabbit anti-claudin-5 antibody (1:100, Affinity, US), rabbit anti-p120-Catenin (1:200, Affinity, US), and rabbit anti- $\beta$-Catenin (1:100, Affinity, US) at $4^{\circ} \mathrm{C}$, and then with Alexa Fluor 488 Affinipure goat anti-rabbit IgG $(\mathrm{H}+\mathrm{L})(1: 200$, Yeasen, China) for $50 \mathrm{~min}$ at room temperature. Phosphatebuffered saline (PBS) was used in place of the primary antibody in the negative control. We use an In Situ Cell Death Detection Kit to detect the apoptotic cells. The nuclei were colored by Hoechst or DAPI. Observation of the fluorescence signal under laser confocal microscopy. Five fields on each of three slides per animal were randomly selected for visualization by light microscopy. Analysis was performed using ImageJ software (National Institutes of Health, Bethesda, MD, USA).

\section{Transmission Electron Microscopy}

After anesthesia by intraperitoneal injection of pentobarbital sodium, the thoracic cavity of rats was opened and the heart was perfused with normal saline. Tissue was quickly removed and cut into $1 \mathrm{~mm}^{3}$ pieces on ice and soaked in $2.5 \%$ glutaraldehyde. The tissue was fixed with a $1 \%$ oxidizing fixative for $1 \mathrm{~h}$ and stained with $1 \%$ uranyl acetate for $2 \mathrm{~h}$, and then the tissue was embedded after dehydration in gradient acetone solution. After semi-thin sectioning and toluidine blue staining, ultrathin sections were cut and observed by Hitachi TEM.

\section{Statistical Analysis}

All experimental data are expressed as the mean \pm standard deviation. Kolmogorov-Smirnov (K-S) test was used for a normality test, $p>0.05$ indicates that it accords with normal distribution; Levene's test was used for a test of homogeneity of variance, $p>0.05$ was considered homogeneity of variance, and vice versa. When comparing the two groups, a t-test was used. One-way ANOVA and Dunnett's test were used to evaluate the data when comparing more than two groups of components. SPSS 16 statistical software for statistical analysis, and $p<0.05$ was considered statistically significant. 


\section{Results}

\section{TT reduces the permeability of BSCB after SCI}

We used an impactor to generate a model of SCI (Fig. 1a). Meanwhile, EB staining and statistical analysis of spinal cord water content were used to detect the function of BSCB. The results showed that the water content increased significantly after SCl, and TT could reduce the oedema caused by SCl (Fig. 1b-c) (M vs. S: $P_{7}<0.001, P_{14}<0.001 ;$ TM vs. M: $\left.P_{7}=0.008, P_{14}<0.001\right)$. The amount of EB exudation increased significantly after SCl compared with that of group S, suggesting BSCB leakage (M vs. S: $\boldsymbol{P}_{\boldsymbol{7}}<\mathbf{0 . 0 0 1}, \boldsymbol{P}_{\mathbf{1 4}}$ $<\mathbf{0 . 0 0 1}$ ). After TT training, infiltration of the BSCB was significantly improved. (Fig. $1 \mathrm{~d}-\mathrm{e}$ ) (TM vs. M: $\boldsymbol{P}_{7}<$ $0.001, P_{14}<0.001$ ). The fluorescence intensity of EB after SCl was much higher than that in the $S$ group. However, the fluorescence level of the TM group was significantly lower than that of the M group (Fig. 1fg) (TM vs. M: $P_{7}<0.001, P_{14}<0.001$ ). All these data indicate that TT can inhibit BSCB disruption.

Fig. 1 TT reduces the permeability of BSCB after $\mathrm{SCl}$. (a) SCI modelwas performed by NYU Impactor (10g $\times 20 \mathrm{~cm}$ ). (b, c) Representative quantification data of spinal cord water content in S M TM groups, columns represent mean $\pm S D, n=5$. (d, e) Represent EB dye permeabilized into spinal cord after $S C l$ and quantification of the amount of Evan's Blue $(\mathrm{ug} / \mathrm{g}), \mathrm{n}=5$. ( $(\mathrm{f}-\mathrm{g})$ Representative fluorescent images of Evans Blue Dye extravasation and quantification of the fluorescence intensity, $n=5 .{ }^{\#} p<0.05$ as $M$ group versus

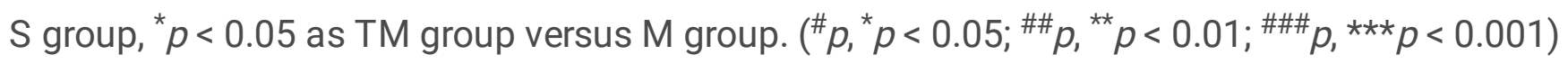

\section{TT decreased tissue structure damage and improved functional recovery after SCl}

At $7 \mathrm{~d}$ and $14 \mathrm{~d}$ after injury, histomorphological differences in T9-T11 levels were observed by HE staining (Fig. 2a). The arrangement of tissues in group $S$ was normal, the grey matter and white matter were destroyed to varying degrees in group $\mathrm{M}$, which was accompanied by the death of multiple neurons (Fig. 2e); the results in group TM was significantly better than that in group $M$. The quantitative analysis of the cavity area showed the same results (Fig. 2b) (TM vs. M: Coronal section, $P_{7}=0.002, P_{14}=0.023$; Sagittal section, $P_{7}=0.008, P_{14}=0.002$ ). The functional recovery of the $\mathrm{S}, \mathrm{M}$ and TM groups was evaluated by BBB scores at $6 \mathrm{~h}, 1 \mathrm{~d}, 3 \mathrm{~d}, 7 \mathrm{~d}$, and $14 \mathrm{~d}$ after SCI (Fig. 2C). The BBB scores of the TM group were significantly higher than those of the $\mathrm{M}$ group at $7 \mathrm{~d}$ and $14 \mathrm{~d}$ after $\mathrm{SCl}$ (TM vs. $\mathrm{M}$ : $\boldsymbol{P}_{\mathbf{7}}=\mathbf{0 . 0 3 6}, \boldsymbol{P}_{\mathbf{1 4}}=\mathbf{0 . 0 1 2}$ ). We further tested the relationship between apoptosis after SCI with TT (Fig. 2d). The number of apoptotic cells in the epicenter increased significantly after SCl. Compared with the M group, the number of apoptotic cells in the TM were significantly decreased (M vs. s: $P<0.001 ;$ TM vs. M: $P=0.007$ ). These results show that TT can significantly improve functional recovery and tissue preservation.

Fig. 2 TT decreased tissue structure damage and improved functional recovery after $\mathrm{SCl}$. (a) HE staining at $7 \mathrm{~d}$ and $14 \mathrm{~d}$ after SCl. Scale bars are $500 \mu \mathrm{m}$. (b) Quantification of the size of cavity area, columns represent mean $\pm S D, n=5$. (c) BBB scores in $S, M$, TM groups. (d) TUNEL staining in the epicenter. (e) Quantitative estimation positive cells. Columns represent mean $\pm S D, n=5 .{ }^{*} p<0.05$ as $M$ group versus $S$ group, ${ }^{*} p<0.05$ as TM group versus M group. $\left({ }^{\#} p,{ }^{*} p<0.05 ;{ }^{\# \#} p,{ }^{* *} p<0.01\right.$; $\left.{ }^{\# \# \#} p,{ }^{* * *} p<0.001\right)$ 


\section{TT prevents the loss of tight junction(TJ) and adhesion junction (AJ) proteins}

To further determine the effect of TT on BSCB protein, AJ protein (B-Catenin, p120-Catenin), TJ protein (Claudin-5, and Occludin) and ZO-1, were examined by Western blot. According to the results (Fig. 3a), the expression of TJ and AJ protein decreased to some extent after SCl compared to the levels of the control (M vs. S: $\beta$-Catenin, p120-Catenin, Claudin-5, Occludin ZO-1, $P<0.001$ ). However, the rats treated with TT showed high TJ and AJ protein expression at $7 \mathrm{~d}$ (Fig. 3b) (TM vs. M: $\beta$-Catenin, $P=0.037$; 1 120-Catenin, $P=0.003$; Claudin-5, $P<0.001$; Occludin, $P=0.004 ; Z 0-1, P<0.001$ ). Using Occludin/CD31/Hoechst (Fig. 3c); Claudin-5/CD31/Hoechst (Fig. 3d); p120-Catenin /CD31/Hoechst (Fig. 4a) and $\beta$-Catenin /CD31/Hoechst (Fig. 4b) staining to observe the distribution of BSCB proteins after SCl, we found that TT could reduce the degradation of Claudin-5/Occludin/p120-Catenin/ $\beta$-Catenin around the epicenter. These results suggest that TT can prevent the loss of TJ and AJ proteins after $\mathrm{SCl}$.

Fig. 3 TT prevent the loss of TJ and AJ protein. $(a, b)$ Represent western blots and quantification data of $T J$ and $A J$ protein in each group, columns represent mean $\pm S D, n=5$. (c-d) Double staining of Occludin/CD31/Hoechst and Claudin-5/CD31/Hoechst. Red: Occludin /Claudin-5; Green: CD31; Blue: Hoechst. Scale bar, $50 \mu \mathrm{m} .{ }^{\#} p<0.05$ as $\mathrm{M}$ group versus $\mathrm{S}$ group, ${ }^{*} p<0.05$ as TM group versus $\mathrm{M}$ group.

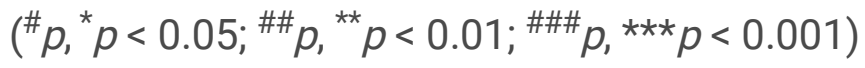

Fig. 4 (a, b) Double staining of p120-Catenin /CD31/Hoechst and $\beta$-Catenin/CD31/Hoechst. Red: p120Catenin/ $\beta$-Catenin; Green: CD31; Blue: Hoechst. Scale bar, $50 \mu \mathrm{m}$. (For interpretation of the references to color in this figure legend, the reader is referred to the web version of this article)

\section{TT promotes angiogenesis after $\mathrm{SCl}$}

We further detected the expression of VEGF protein $7 \mathrm{~d}$ in the ischemic penumbra (The shaded part in Fig. 5a) after $\mathrm{SCl}$. Compared with its expression in group $\mathrm{S}$, the expression of VEGF decreased significantly after SCI (M vs. S: $\boldsymbol{P}=\mathbf{0 . 0 2 9}$ ). At the same time, TT significantly upregulated the expression of VEGF (Fig. 5b-c) (TM vs. M: $P=0.039$ ). Blood vessels that co-labeled with 5 -bromo-2-deoxyuridine ( $\mathrm{BrdU}$ ) and laminin (Laminin) in spinal cord tissue were quantitatively analysed. As shown in Fig. $\mathbf{5 d - e}$, angiogenesis in the M group was significantly higher than it was in the $S$ group at $7 \mathrm{~d}$ after injury ( $M$ vs. $S: P<0.001$ ).

Additionally, the neovascularization density of the TM group was increased compared with that in the $M$ group, suggesting that TT can effectively promote angiogenesis (TM vs. $M$ : $P<0.001$ ).

Fig. 5 TT promotes angiogenesis after SCl. (a) Schematic diagram of sampling and positioning (b, c) Representative western blots and quantification data of VEGF/Tubulin, columns represent mean $\pm S D$, $\mathrm{n}=5$. (e) Double staining of Laminin(green)/Brdu (red) of sections from the spinal cord in each group rats. Scale bars are $20 \mu \mathrm{m}$. (d) Quantification data of number of Brdu and Laminin co-stained cells, columns represent mean $\pm \mathrm{SD}, \mathrm{n}=5$. ${ }^{\#} p<0.05$ as $\mathrm{M}$ group versus $\mathrm{S}$ group, ${ }^{*} p<0.05$ as $\mathrm{TM}$ group versus $\mathrm{M}$ group.

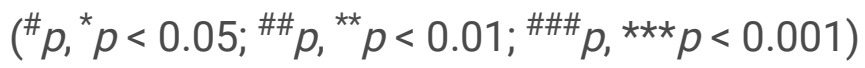




\section{TT inhibits the expression of MMP-2/9 after SCI}

The expression level of MMP-2/9 protein was detected by Western blot. The results showed that TT could significantly inhibit the upregulation of MMP-2/9 after SCI (Fig. 6a-c) (TM vs. M: MMP-2, $P<0.001$; MMP9, $P=0.002$ ). In addition, to determine the defects of vascular ECs, we analysed them by TEM. In group $S$, the vascular ECs of spinal cord showed a tight connection of rivet-like structure, and a series of electronic dense bands appeared between the plasma membranes, forming a relatively closed vascular lumen, and the intercellular TJ was partially opened after SCl. Compared with group S in the same period, the structure of BSCB in group M was disordered, the electron dense zone of TJ was lighter, the ECs were atrophied and scar tissue was formed around the blood vessels. On the contrary, the rats in the TM group had more electronic dense bands, and the ultrastructural changes were between S and M (Fig. 6d).

Fig. 6 TT inhibits the expression of MMP-2 and MMP-9 after SCI. (a-c) Represent western blots and quantification data of MMP-2/9 in each group, columns represent mean $\pm S D, n=5$. ${ }^{\#} p<0.05$ as $M$ group versus $S$ group, ${ }^{*} p<0.05$ as TM group versus $M$ group. (d) TEM showed the EC-EC junctions in $S, M, T M$. Arrows indicate TJ electron dense band and the open TJ, scale bars are $1.0 \mu \mathrm{m} .\left({ }^{\#} p,{ }^{*} p<0.05 ;{ }^{\# \#} p,{ }^{* \star} p<\right.$ $\left.0.01 ;{ }^{\# \# \#} p,{ }^{\star \star *} p<0.001\right)$

\section{Discussion}

In this study, our data indicate that TT can protect the integrity of BSCB and prevent further edema of spinal cord. After TT training, the organizational structure and motor function can also be significantly improved. Meanwhile, TT can promote angiogenesis, reduce apoptosis and inhibit the expression of MMP-2/9. Therefore, the vascular protection of the BSCB by TT may occur through the following mechanisms: (1) TT protects residual BSCB structure from further damage; (2) it promotes vascular regeneration; and (3) it inhibits the expression of MMP-2/9 to mitigate BSCB damage.

Emerging evidence indicates that exercise therapy can promote recovery after SCI [31-34]. It is well known that normal exercise training cannot be carried out in rats after $\mathrm{SCl}$. We introduced water treadmill training(TT), which simulates the clinical exercise treatment and can help rats successfully train on the treadmill in the early stage of $\mathrm{SCl}$. TT is mainly used in rehabilitation training of motor system diseases $[35,36]$. TT can reduce the resistance of forward movement, allowing patients to walk or run with a normal gait [37]. Additionally, the depth and speed of the TT can be adjusted, which facilitates the control of exercise intensity [37], and its related mechanism was studied for the first time in our experiment.

Trauma, infection, tumor growth or obstruction of blood supply can cause edema of the central nervous system (CNS). Cytotoxicity and vasogenic edema are the main interdependent factors in the development of CNS edema. The prolongation of cytotoxic edema leads to vasogenic edema, and vice versa[38, 39]. Cytotoxic edema refers to the accumulation of water in intact cells. When an anoxic state leads to the loss of energy-dependent solute dynamic balance, it triggers quickly [40]. The accumulation of fluid through the extracellular space of damaged BBB/BSCB is called vasogenic edema [41]. The integrity of 
the BSCB is essential for the spinal cord to maintain normal function [42]. However, the BSCB is destroyed after $\mathrm{SCl}$, which leads to increased permeability, causing a series of secondary damage [18, 43]. In our study, through the horizontal comparison of data of each group in the same period, we found that the degree of edema in the $M$ group increased significantly compared to that of $S$ group, accompanied by lower BBB scores, severe tissue structure damage and a large number of cell necrosis. However, these conditions have been significantly improved after TT training, this may be related to the protective effect of TT on BSCB. By longitudinal comparison of the BBB scores $7 \mathrm{~d}$ and $14 \mathrm{~d}$ after $\mathrm{SCl}$ in the $\mathrm{M}$ group, it was found that there was some amount of self-recovery. The recovery of these partial functions may be related to the preservation of BSCB function, and the formation of new blood vessels.

The BSCB protects the central nervous system by restricting the entry of plasma components and blood cells [12]. Following SCl, the destruction of the BSCB leads to increased microvascular permeability, inflammatory reaction, tissue edema, and neurotoxic products [44]. The BSCB is formed by a dense network of TJs and AJs, which is destroyed after SCl, resulting in a decrease in the expression of $\mathrm{TJ}$ and AJ protein. Tight, adhesion, and gap junctions form the endothelial cells lining of microvessels in the spinal cord[3, 45]. Our results show that the expression of p120-Catenin, $\beta$-Catenin, ZO-1, Occludin, and Claudin- 5 were greatly reduced after SCl. However, their expressions were significantly stabilized in the TT-treated spinal cord. This shows that the residual BSCB structure is protected from being destroyed, which may be a mechanism by which TT plays a protective role.

In order to determine the protection mechanism of TT, we have carried out more in-depth research. Vascular endothelial growth factor (VEGF) stimulates the proliferation and survival of endothelial cells, the formation of vascular structures, nitric-oxide dependent vasodilatation and vascular leakage [17]. It has been reported that VEGF reached a peak at $3 d$, and there was still a large amount of expression at 7 $\mathrm{d}$ after $\mathrm{SCl}$ [46]. In addition, ischemia and injury can induce angiogenesis, which will provide oxygen and nutrition to the ischemic or diseased site, thus improving tissue repair and remodeling [47-49]. We used laminin as a vascular marker and BrdU as a proliferation marker [50]. The results showed that TT could promote angiogenesis after $\mathrm{SCl}$, as shown by detecting the protein expression of VEGF. Quantitative analysis of neovascularization $7 \mathrm{~d}$ after $\mathrm{SCl}$ with co-labelling also showed that the number of $\mathrm{BrdU}^{+} /$Laminin $^{+}$cells increased significantly after TT treatment in rats with $\mathrm{SCl}$, which will may help to maintain the stability of BSCB.

The activation of MMP-2/9 after SCI plays an essential role in the destruction of the BBB/BSCB $[9,12]$. The expression of MMP-2/9 will aggravate the damage of BSCB[51]. In this study, $7 \mathrm{~d}$ after SCl, the expressions of MMP-2/9 were up-regulated, which was due to physiological self-regulation after SCI. But this further aggravates the damage to BSCB. We were surprised to find that the expression of MMP-2/9 decreased significantly after TT training. Although we have not proved how TT down-regulates the expression of MMP-2/9, we believe that TT can effectively prevent the destruction of the BSCB, and part of its mechanism may be to inhibit the expression of MMP-2/9 after SCl. 
As far as we know, this is the first time that we have applied TT to the treatment of SCl. We found that TT can enhance the expression of TJ and AJ proteins after SCl. We also found that TT could promote angiogenesis and inhibit the expression of MMP-2/9, which may be an important mechanism for TT to maintain the stability of BSCB. In addition, these experimental results will provide a better understanding of the possible mechanism of TT in the treatment of $\mathrm{SCl}$ and provide a reliable basis for TT application in the future.

We acknowledge the limitations of our studies. In this experiment, we only used male rats, ignoring the possible differences caused by sex. Due to the limitation of experimental conditions, we could not observe changes at the functional level through electrophysiological techniques to understand the role of TT. The training period was relatively short and the specific mechanism by which TT inhibits the expression of MMP-2/9 remains to be further studied.

Fig. 7 Proposed mechanism for the BSCB protection of water treadmill training after SCI.

\section{Conclusion}

TT participates in protection of the BSCB after SCI by protects residual BSCB structure from further damage. Furthermore, TT promotes vascular regeneration. Additionally, TT Inhibits the expression of MMP-2/9 to mitigate BSCB damage (Fig. 7). However, TT-induced BSCB protection after SCl is a complex, a cascade of signaling events and multi-channel process that involves many key factors and remains to be fully elucidated. As mentioned in the limitations of our discussion, our next research direction will be an in-depth study of the upstream molecular mechanisms of MMP-2/9 and VEGF, using techniques such as in vivo electrophysiology to study at the functional level.

\section{Abbreviations}

SCl, Spinal cord injury; TT, Water treadmill training; BSCB, Blood-spinal cord barrier; TJ, tight junction; AJ, adhesion junction; MMP, Matrix metalloproteinase; BBB, Basso-Beattie-Bresnahan; HE, Haematoxylineosin; EB, Evans blue; WB, Western blot; TEM, Transmission electron microscopy; VEGF, Vascular endothelial growth factor; PBS, Phosphate-buffered saline; BrdU, 5-bromo-2-deoxyuridine; EC, Endothelial cell; CD31: Platelet endothelial cell adhesion molecule-1; CNS, Central nervous system.

\section{Declarations}

\section{Ethics approval and consent to participate}

The protocols were approved by the Animal Research Committee of Wenzhou Medical University

\section{Consent for publication}

Not applicable 
Availability of data and materials

The datasets supporting the conclusions of this article are available from the corresponding author on reasonable request

\section{Competing interests}

The authors declare that they have no competing interests.

\section{Funding}

This research was partly supported by the National Natural Science Foundation of China (No.81873376, 81802243, 81603433, 81574074).

\section{Authors' contributions}

YXW designed the experiments and wrote the first manuscript; XQF and LSC was responsible for data acquisition and analysis; YXL, ZKC, YJJ and CXL conceived the experiments; TWZ and YGH provided guidance; JSH supervised the whole process of the experiment, and was a major contributor in writing the manuscript. All authors read and approved the final manuscript.

\section{Acknowledgements}

We would like to express our heartfelt thanks to all the technical personnel who have provided assistance in this work.

\section{References}

1. Cordaro, M., et al., Fumaric Acid Esters Attenuate Secondary Degeneration after Spinal Cord Injury. J Neurotrauma, 2017. 34(21): p. 3027-3040.

2. Rao, J., R. Tiruchelvarayan, and L. Lee, Palliative surgery for cervical spine metastasis. Singapore Med J, 2014. 55(11): p. 569-73.

3. Bartanusz, V., et al., The blood-spinal cord barrier: morphology and clinical implications. Ann Neurol, 2011. 70(2): p. 194-206.

4. Zheng, B., et al., Epidermal growth factor attenuates blood-spinal cord barrier disruption via PI3K/Akt/Rac1 pathway after acute spinal cord injury. J Cell Mol Med, 2016. 20(6): p. 1062-75.

5. Beck, K.D., et al., Quantitative analysis of cellular inflammation after traumatic spinal cord injury: evidence for a multiphasic inflammatory response in the acute to chronic environment. Brain, 2010. 133(Pt 2): p. 433-47.

6. Abbott, N.J., L. Ronnback, and E. Hansson, Astrocyte-endothelial interactions at the blood-brain barrier. Nat Rev Neurosci, 2006. 7(1): p. 41-53. 
7. Yao, Y., et al., Flufenamic acid inhibits secondary hemorrhage and BSCB disruption after spinal cord injury. Theranostics, 2018. 8(15): p. 4181-4198.

8. Zhang, D., et al., Metformin ameliorates BSCB disruption by inhibiting neutrophil infiltration and MMP-9 expression but not direct TJ proteins expression regulation. 2017. 21(12): p. 3322-3336.

9. Asahi, M., et al., Effects of matrix metalloproteinase-9 gene knock-out on the proteolysis of bloodbrain barrier and white matter components after cerebral ischemia. J Neurosci, 2001. 21(19): p. 7724-32.

10. Heo, J.H., et al., Matrix metalloproteinases increase very early during experimental focal cerebral ischemia. J Cereb Blood Flow Metab, 1999. 19(6): p. 624-33.

11. Chang, D.I., et al., Activation systems for latent matrix metalloproteinase-2 are upregulated immediately after focal cerebral ischemia. J Cereb Blood Flow Metab, 2003. 23(12): p. 1408-19.

12. Noble, L.J., et al., Matrix metalloproteinases limit functional recovery after spinal cord injury by modulation of early vascular events. J Neurosci, 2002. 22(17): p. 7526-35.

13. Lee, J.Y., et al., 17beta-estradiol inhibits MMP-9 and SUR1/TrpM4 expression and activation and thereby attenuates BSCB disruption/hemorrhage after spinal cord injury in male rats. Endocrinology, 2015. 156(5): p. 1838-50.

14. Xie, Q., et al., Treadmill exercise ameliorates focal cerebral ischemia/reperfusion-induced neurological deficit by promoting dendritic modification and synaptic plasticity via upregulating caveolin-1/VEGF signaling pathways. Exp Neurol, 2019. 313: p. 60-78.

15. Brockington, A., et al., Vascular endothelial growth factor and the nervous system. Neuropathol Appl Neurobiol, 2004. 30(5): p. 427-46.

16. Rosenstein, J.M. and J.M. Krum, New roles for VEGF in nervous tissue--beyond blood vessels. Exp Neurol, 2004. 187(2): p. 246-53.

17. Ferrara, N., H.P. Gerber, and J. LeCouter, The biology of VEGF and its receptors. Nat Med, 2003. 9(6): p. 669-76.

18. Lu, Y., et al., Bone Mesenchymal Stem Cell-Derived Extracellular Vesicles Promote Recovery Following Spinal Cord Injury via Improvement of the Integrity of the Blood-Spinal Cord Barrier. Front Neurosci, 2019. 13: p. 209.

19. Park, C.S., et al., Protocatechuic acid improves functional recovery after spinal cord injury by attenuating blood-spinal cord barrier disruption and hemorrhage in rats. Neurochem Int, 2019. 124: p. 181-192.

20. Leech, K.A. and T.G. Hornby, High-Intensity Locomotor Exercise Increases Brain-Derived Neurotrophic Factor in Individuals with Incomplete Spinal Cord Injury. J Neurotrauma, 2017. 34(6): p. 1240-1248.

21. Ward, P.J., et al., Training-Induced Functional Gains following SCI. Neural Plast, 2016. 2016: p. 4307694.

22. Wang, $\mathrm{H}$., et al., Treadmill training induced lumbar motoneuron dendritic plasticity and behavior recovery in adult rats after a thoracic contusive spinal cord injury. Exp Neurol, 2015. 271: p. 368-78. 
23. Hayashibe, M., et al., Locomotor improvement of spinal cord-injured rats through treadmill training by forced plantar placement of hind paws. Spinal Cord, 2016. 54(7): p. 521-9.

24. Sharma, H.S., et al., Neuroprotective effects of a potent bradykinin B2 receptor antagonist HOE-140 on microvascular permeability, blood flow disturbances, edema formation, cell injury and nitric oxide synthase upregulation following trauma to the spinal cord. Int Rev Neurobiol, 2019. 146: p. 103-152.

25. Sahib, S., et al., Potentiation of spinal cord conduction and neuroprotection following nanodelivery of DL-3-n-butylphthalide in titanium implanted nanomaterial in a focal spinal cord injury induced functional outcome, blood-spinal cord barrier breakdown and edema formation. Int Rev Neurobiol, 2019. 146: p. 153-188.

26. Ying, X., et al., Hyperbaric oxygen therapy reduces apoptosis and dendritic/synaptic degeneration via the BDNF/TrkB signaling pathways in SCl rats. Life Sci, 2019. 229: p. 187-199.

27. Basso, D.M., M.S. Beattie, and J.C. Bresnahan, $A$ sensitive and reliable locomotor rating scale for open field testing in rats. J Neurotrauma, 1995. 12(1): p. 1-21.

28. Lv, R., et al., Polydatin attenuates spinal cord injury in rats by inhibiting oxidative stress and microglia apoptosis via Nrf2/HO-1 pathway. Life Sci, 2019. 217: p. 119-127.

29. Saadoun, S., et al., Greatly improved neurological outcome after spinal cord compression injury in AQP4-deficient mice. Brain, 2008. 131(Pt 4): p. 1087-98.

30. Berliner, J., et al., Abnormalities in spinal cord ultrastructure in a rat model of post-traumatic syringomyelia. Fluids Barriers CNS, 2020. 17(1): p. 11.

31. Dugan, E.A., S. Jergova, and J. Sagen, Mutually beneficial effects of intensive exercise and GABAergic neural progenitor cell transplants in reducing neuropathic pain and spinal pathology in rats with spinal cord injury. Exp Neurol, 2020. 327: p. 113208.

32. Kobayakawa, K., et al., Locomotor Training Increases Synaptic Structure With High NGL-2 Expression After Spinal Cord Hemisection. Neurorehabil Neural Repair, 2019. 33(3): p. 225-231.

33. Kissane, R.W.P., et al., Effects of treadmill training on microvascular remodeling in the rat after spinal cord injury. 2019. 59(3): p. 370-379.

34. Ryu, Y., et al., Effects of Treadmill Training Combined with Serotonergic Interventions on Spasticity after Contusive Spinal Cord Injury. J Neurotrauma, 2018. 35(12): p. 1358-1366.

35. Ayan, C., et al., Treadmill training combined with water and land-based exercise programs: Effects on Parkinson's disease patients. NeuroRehabilitation, 2016. 39(2): p. 295-9.

36. Narasaki-Jara, M., et al., Aquatic treadmill walking at three depths of water in people with traumatic brain injury. Physiother Res Int, 2020. 25(2): p. e1817.

37. Munoz, A., et al., The Use of the Water Treadmill for the Rehabilitation of Musculoskeletal Injuries in the Sport Horse. J Vet Res, 2019. 63(3): p. 439-445.

38. Jha, R.M., P.M. Kochanek, and J.M. Simard, Pathophysiology and treatment of cerebral edema in traumatic brain injury. Neuropharmacology, 2019. 145(Pt B): p. 230-246. 
39. Kitchen, P., et al., Targeting Aquaporin-4 Subcellular Localization to Treat Central Nervous System Edema. Cell, 2020. 181(4): p. 784-799.e19.

40. Bordone, M.P. and M.M. Salman, The energetic brain - A review from students to students. 2019. 151(2): p. 139-165.

41. Simard, J.M., et al., Brain oedema in focal ischaemia: molecular pathophysiology and theoretical implications. Lancet Neurol, 2007. 6(3): p. 258-68.

42. Zheng, B., et al., Dl-3-n-butylphthalide prevents the disruption of blood-spinal cord barrier via inhibiting endoplasmic reticulum stress following spinal cord injury. Int J Biol Sci, 2017. 13(12): p. 1520-1531.

43. He, Z., et al., Inhibition of Endoplasmic Reticulum Stress Preserves the Integrity of Blood-Spinal Cord Barrier in Diabetic Rats Subjected to Spinal Cord Injury. 2017. 7(1): p. 7661.

44. Yang, Y., et al., Matrix metalloproteinase-mediated disruption of tight junction proteins in cerebral vessels is reversed by synthetic matrix metalloproteinase inhibitor in focal ischemia in rat. J Cereb Blood Flow Metab, 2007. 27(4): p. 697-709.

45. Joshi, H.P., et al., CORM-2-Solid Lipid Nanoparticles Maintain Integrity of Blood-Spinal Cord Barrier After Spinal Cord Injury in Rats. Mol Neurobiol, 2020. 57(6): p. 2671-2689.

46. Yu, D., et al., [Effects of bone marrow mesenchymal stem cells transplantation on expression of vascular endothelial growth factor gene and angiogenesis after spinal cord injury in rats]. Zhongguo Xiu Fu Chong Jian Wai Ke Za Zhi, 2011. 25(7): p. 837-41.

47. Loh, K.P., et al., TRPM4 inhibition promotes angiogenesis after ischemic stroke. Pflugers Arch, 2014. 466(3): p. 563-76.

48. Zhu, W., et al., Insulin growth factor-1 gene transfer enhances neurovascular remodeling and improves long-term stroke outcome in mice. Stroke, 2008. 39(4): p. 1254-61.

49. Huang, X.T., et al., Intracerebroventricular transplantation of ex vivo expanded endothelial colonyforming cells restores blood-brain barrier integrity and promotes angiogenesis of mice with traumatic brain injury. J Neurotrauma, 2013. 30(24): p. 2080-8.

50. Pang, Q., et al., Role of caveolin-1/vascular endothelial growth factor pathway in basic fibroblast growth factor-induced angiogenesis and neurogenesis after treadmill training following focal cerebral ischemia in rats. Brain Res, 2017. 1663: p. 9-19.

51. Jing, N., et al., Exogenous activation of cannabinoid-2 receptor modulates TLR4/MMP9 expression in a spinal cord ischemia reperfusion rat model. J Neuroinflammation, 2020. 17(1): p. 101.

\section{Figures}




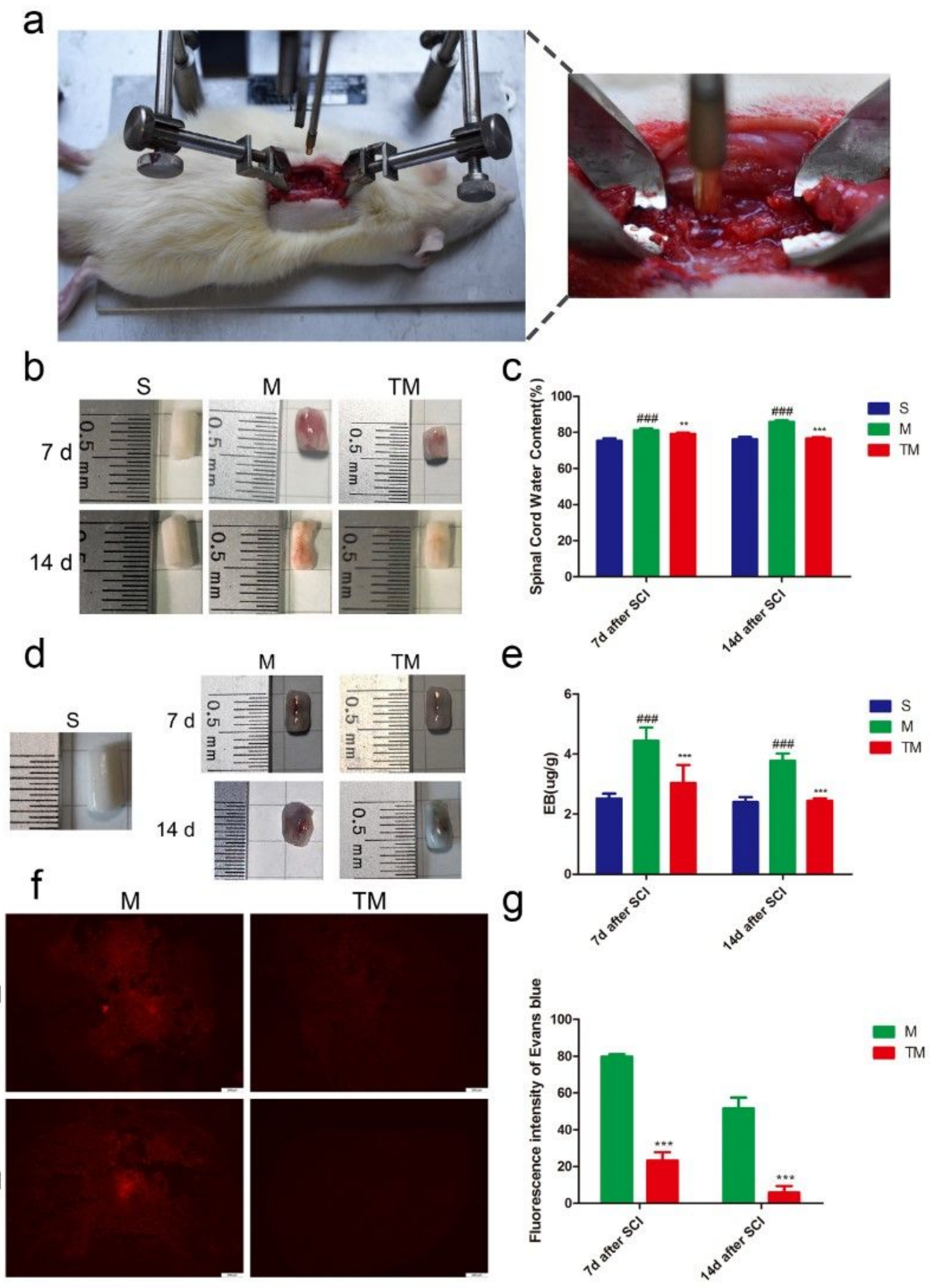

Figure 1

TT reduces the permeability of BSCB after SCl. (a) SCI modelwas performed by NYU Impactor $(10 \mathrm{~g} \times$ $20 \mathrm{~cm}$ ). (b, c) Representative quantification data of spinal cord water content in S M TM groups, columns represent mean $\pm S D, n=5$. (d, e) Represent EB dye permeabilized into spinal cord after $S C l$ and quantification of the amount of Evan's Blue $(\mathrm{ug} / \mathrm{g}), \mathrm{n}=5$. (f-g) Representative fluorescent images of Evans 
Blue Dye extravasation and quantification of the fluorescence intensity, $n=5$. \#p $<0.05$ as $M$ group versus S group, * $\mathrm{p}<0.05$ as TM group versus M group. (\#p, ${ }^{*} p<0.05 ; \# \# p,{ }^{* *} p<0.01 ; \# \# \# p,{ }^{* *} p<0.001$ )

a
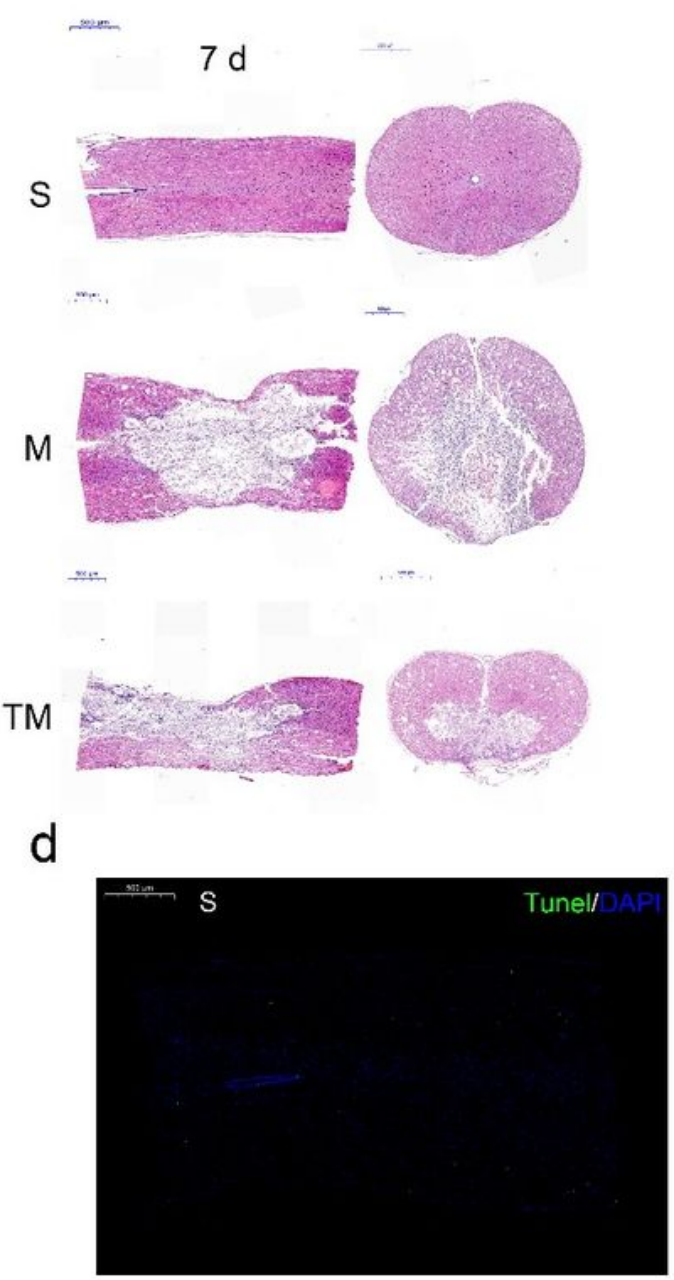

e

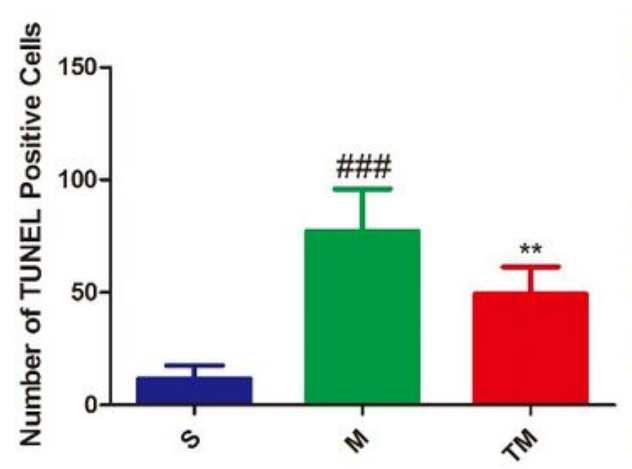

b

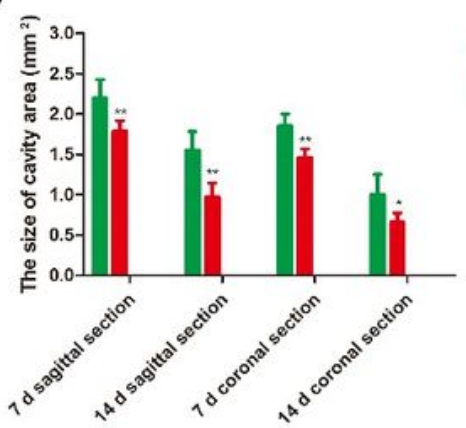

C

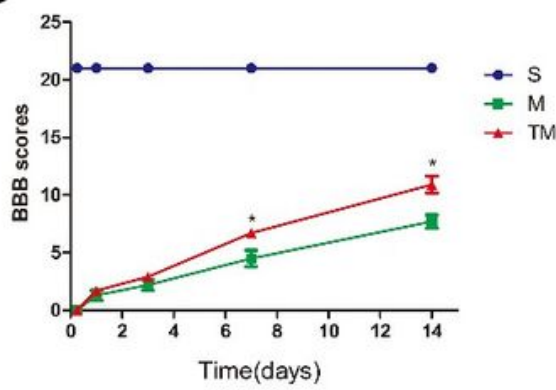

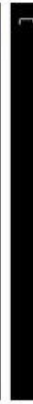
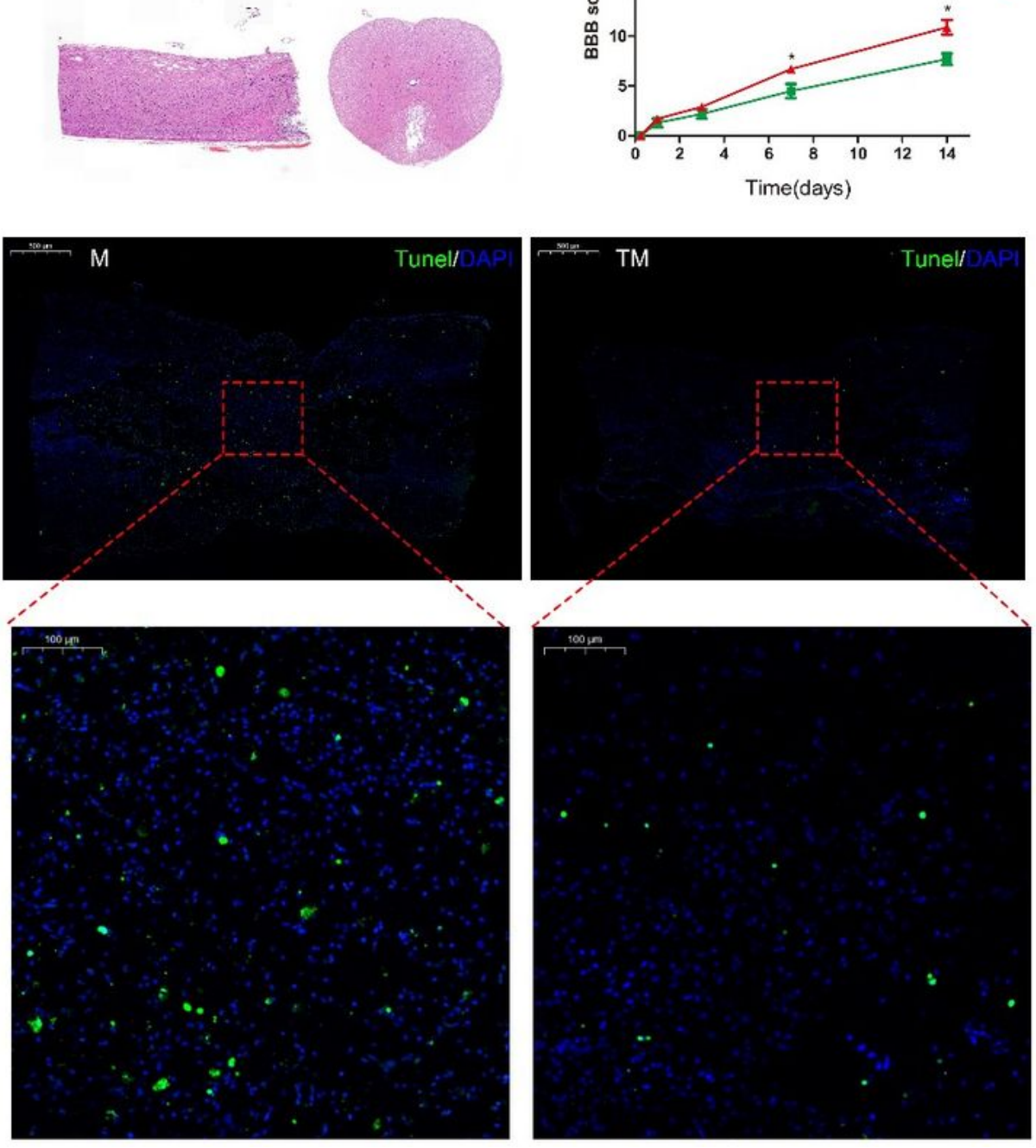

Figure 2

TT decreased tissue structure damage and improved functional recovery after SCl. (a) HE staining at $7 \mathrm{~d}$ and $14 \mathrm{~d}$ after SCl. Scale bars are $500 \mu \mathrm{m}$. (b) Quantification of the size of cavity area, columns represent mean \pm SD, $n=5$. (c) BBB scores in S, M, TM groups. (d) TUNEL staining in the epicenter. (e) Quantitative 
estimation positive cells. Columns represent mean $\pm \mathrm{SD}, \mathrm{n}=5$. \#p $<0.05$ as $\mathrm{M}$ group versus $\mathrm{S}$ group, *p $<$ 0.05 as TM group versus M group. (\#p, *p<0.05; \#\#p, **p<0.01; \#\#\#p, **p <0.001)

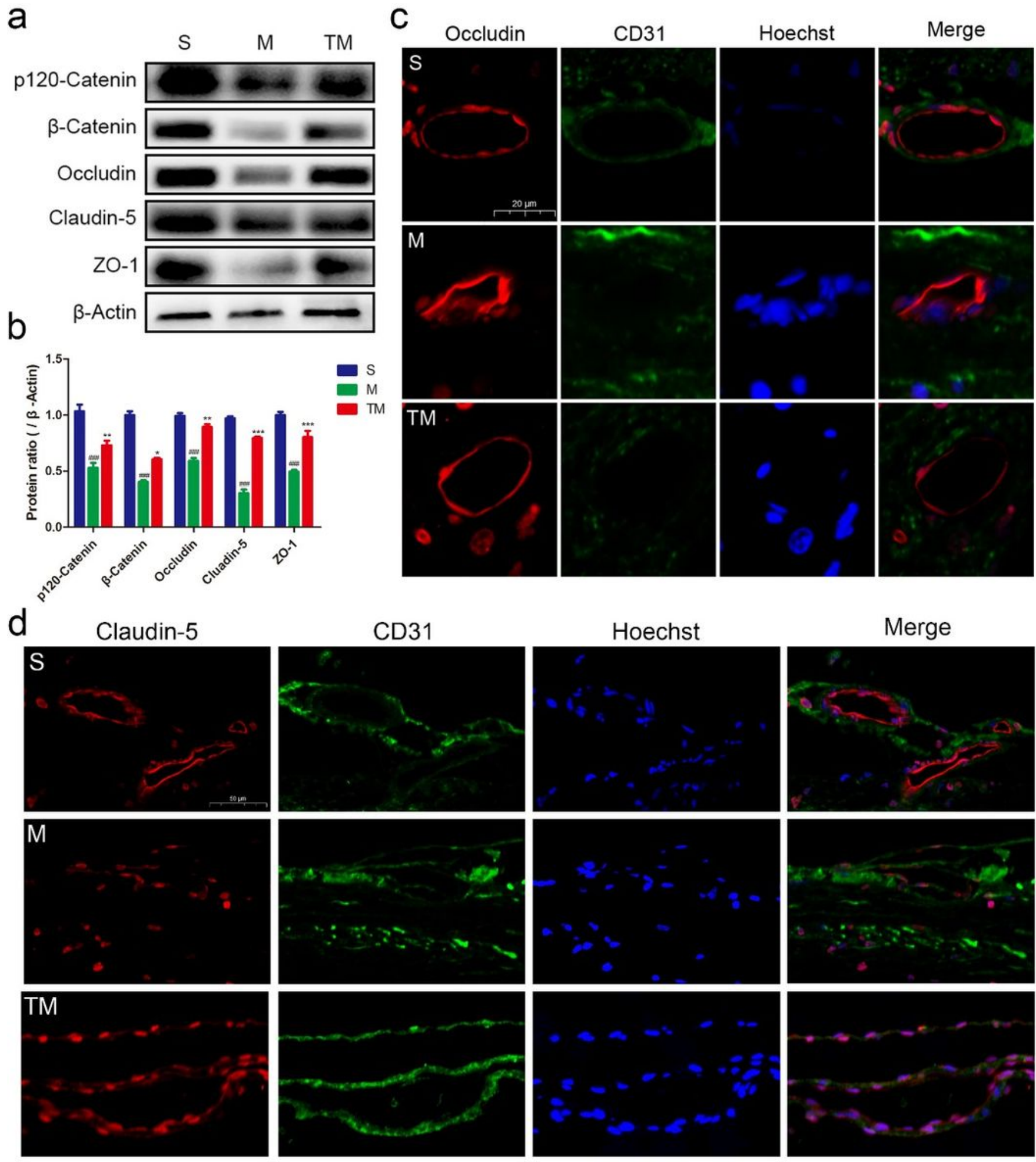

\section{Figure 3}

TT prevent the loss of TJ and AJ protein. $(a, b)$ Represent western blots and quantification data of TJ and AJ protein in each group, columns represent mean $\pm S D, n=5$. (c-d) Double staining of Occludin/CD31/Hoechst and Claudin-5/CD31/Hoechst. Red: Occludin /Claudin-5; Green: CD31; Blue: 
Hoechst. Scale bar, $50 \mu \mathrm{m}$. \#p $<0.05$ as $M$ group versus $S$ group, ${ }^{\star} p<0.05$ as TM group versus $M$ group. $\left(\# p,{ }^{*}<0.05 ; \# \# p, * * p<0.01 ; \# \# \#\right.$, ***p $\left.<0.001\right)$
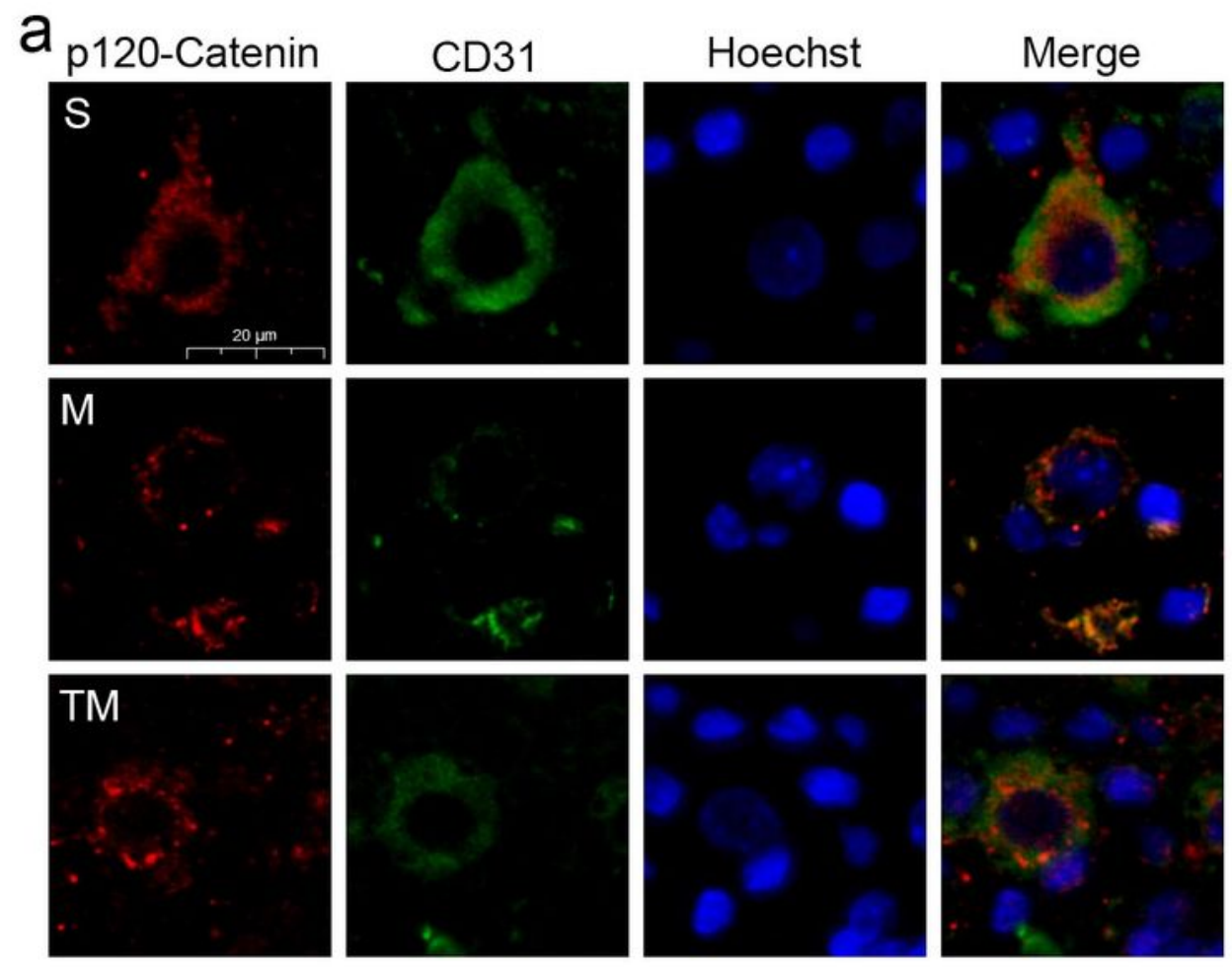

b
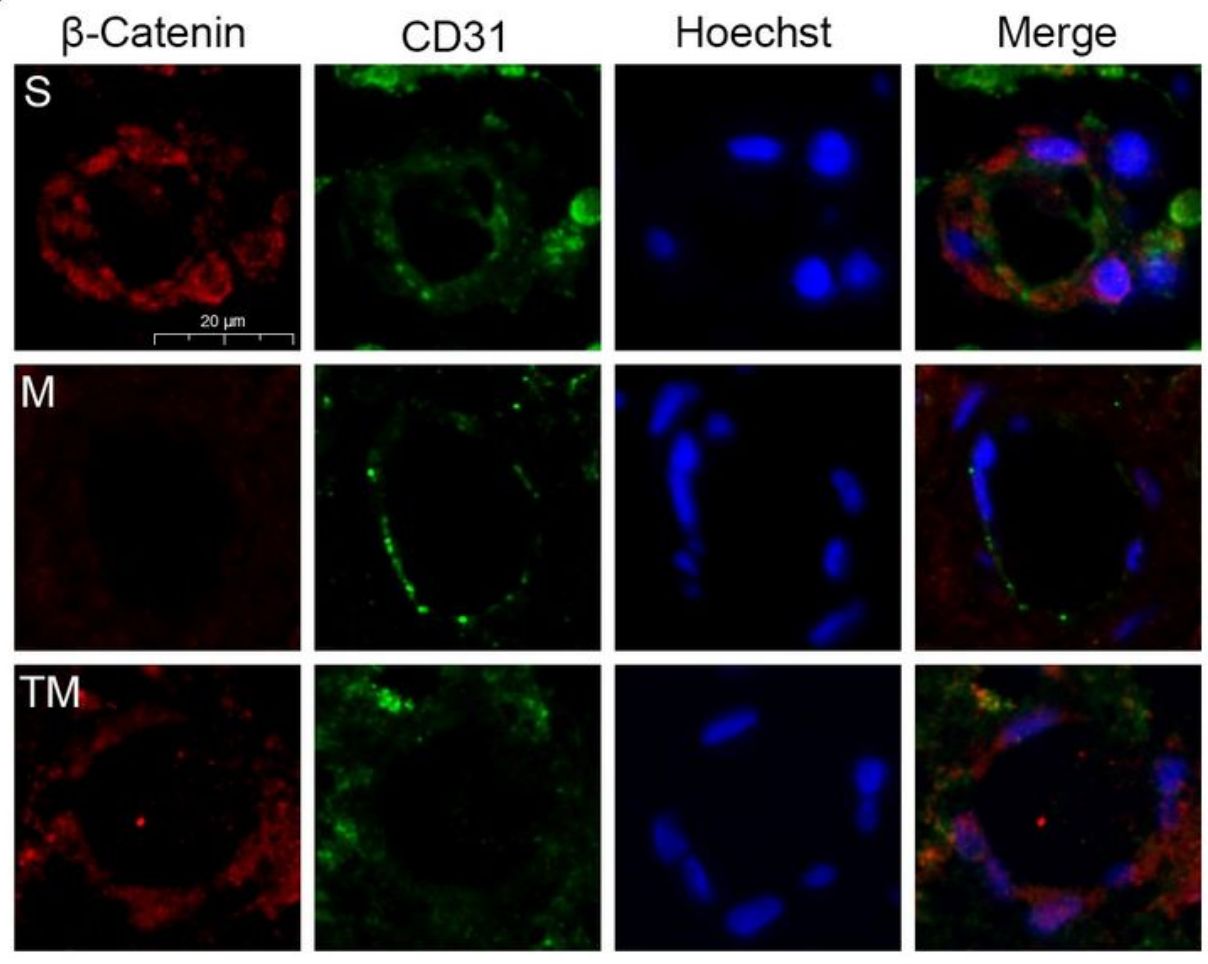

Figure 4

$(a, b)$ Double staining of p120-Catenin /CD31/Hoechst and $\beta$-Catenin/CD31/Hoechst. Red: p120-Catenin/ $\beta$-Catenin; Green: CD31; Blue: Hoechst. Scale bar, $50 \mu \mathrm{m}$. (For interpretation of the references to color in this figure legend, the reader is referred to the web version of this article) 

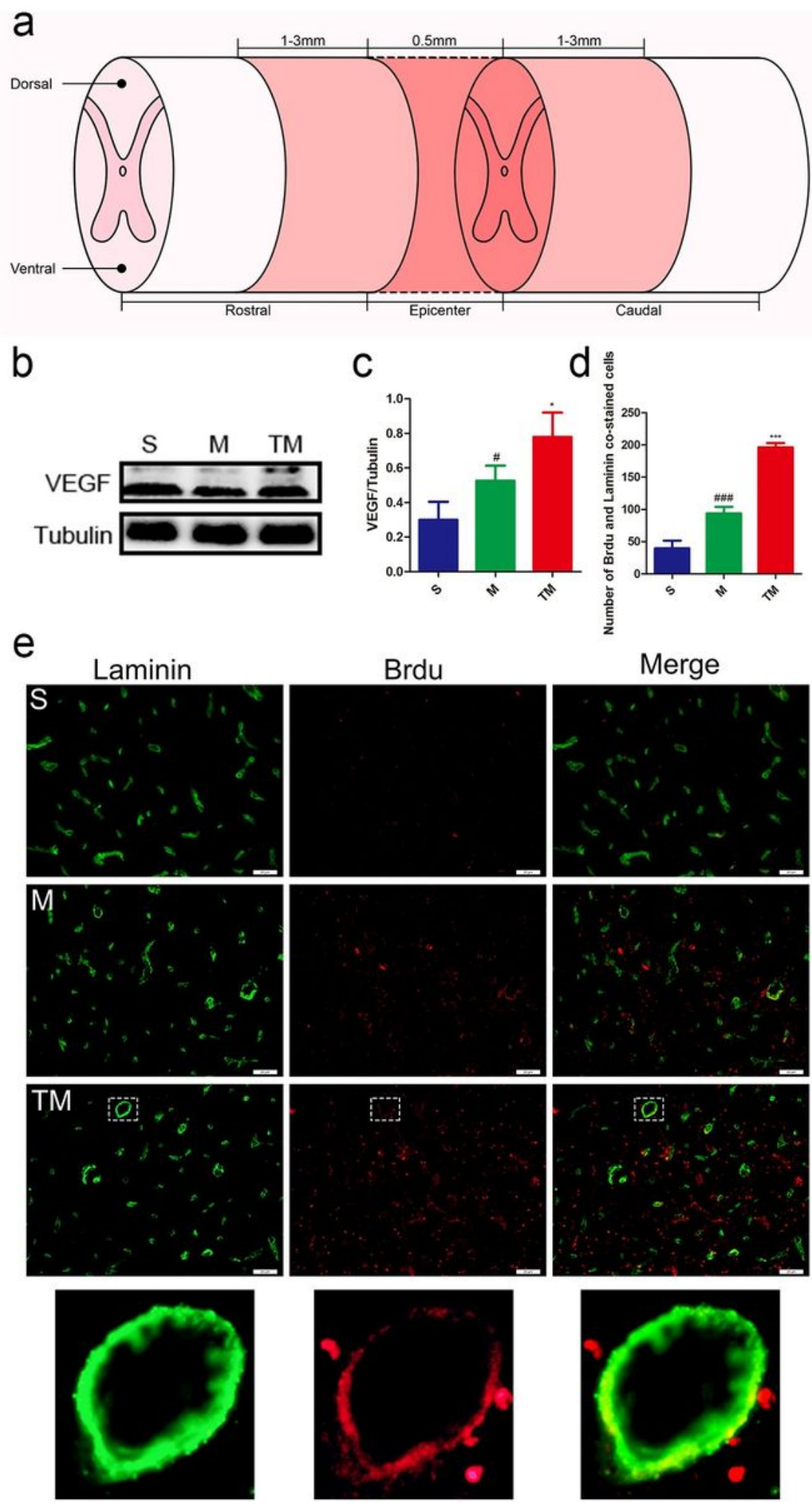

\section{Figure 5}

TT promotes angiogenesis after $\mathrm{SCl}$. (a) Schematic diagram of sampling and positioning (b, c) Representative western blots and quantification data of VEGF/Tubulin, columns represent mean $\pm S D$, $\mathrm{n}=5$. (e) Double staining of Laminin(green)/Brdu (red) of sections from the spinal cord in each group rats. Scale bars are $20 \mu \mathrm{m}$. (d) Quantification data of number of Brdu and Laminin co-stained cells, columns 
represent mean $\pm S D, n=5$. \#p $<0.05$ as $M$ group versus $S$ group, ${ }^{\star} p<0.05$ as $T M$ group versus $M$ group. $\left(\# p,{ }^{*}<<0.05 ; \# \# p,{ }^{* *} p<0.01 ; \# \# \# p, * * * p<0.001\right)$

a

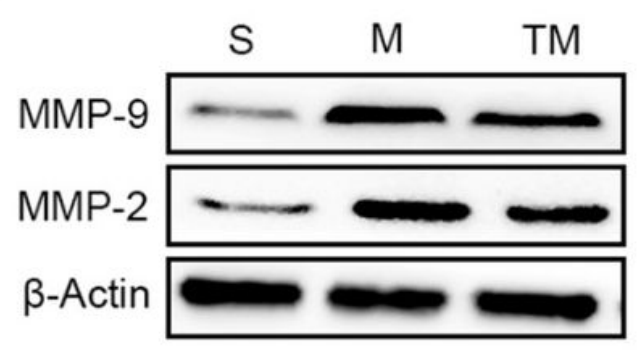

d

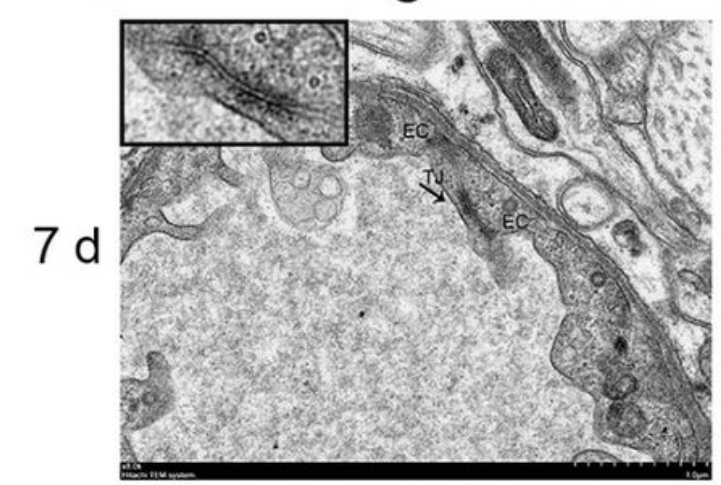

$14 \mathrm{~d}$

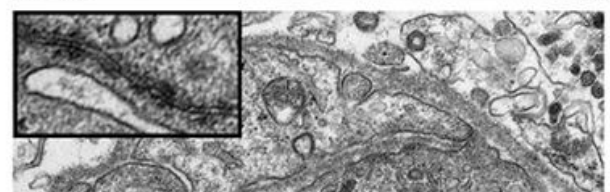

b

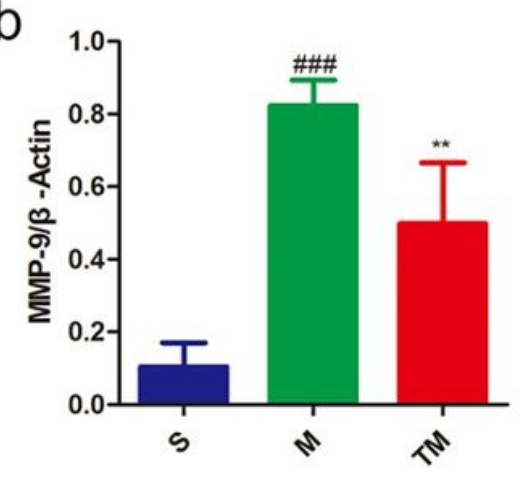

$\mathrm{M}$

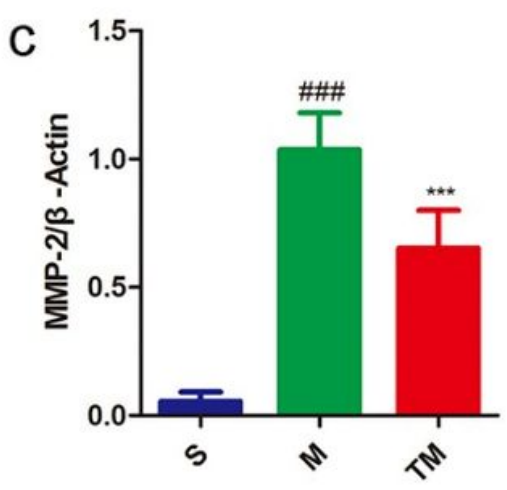

TM
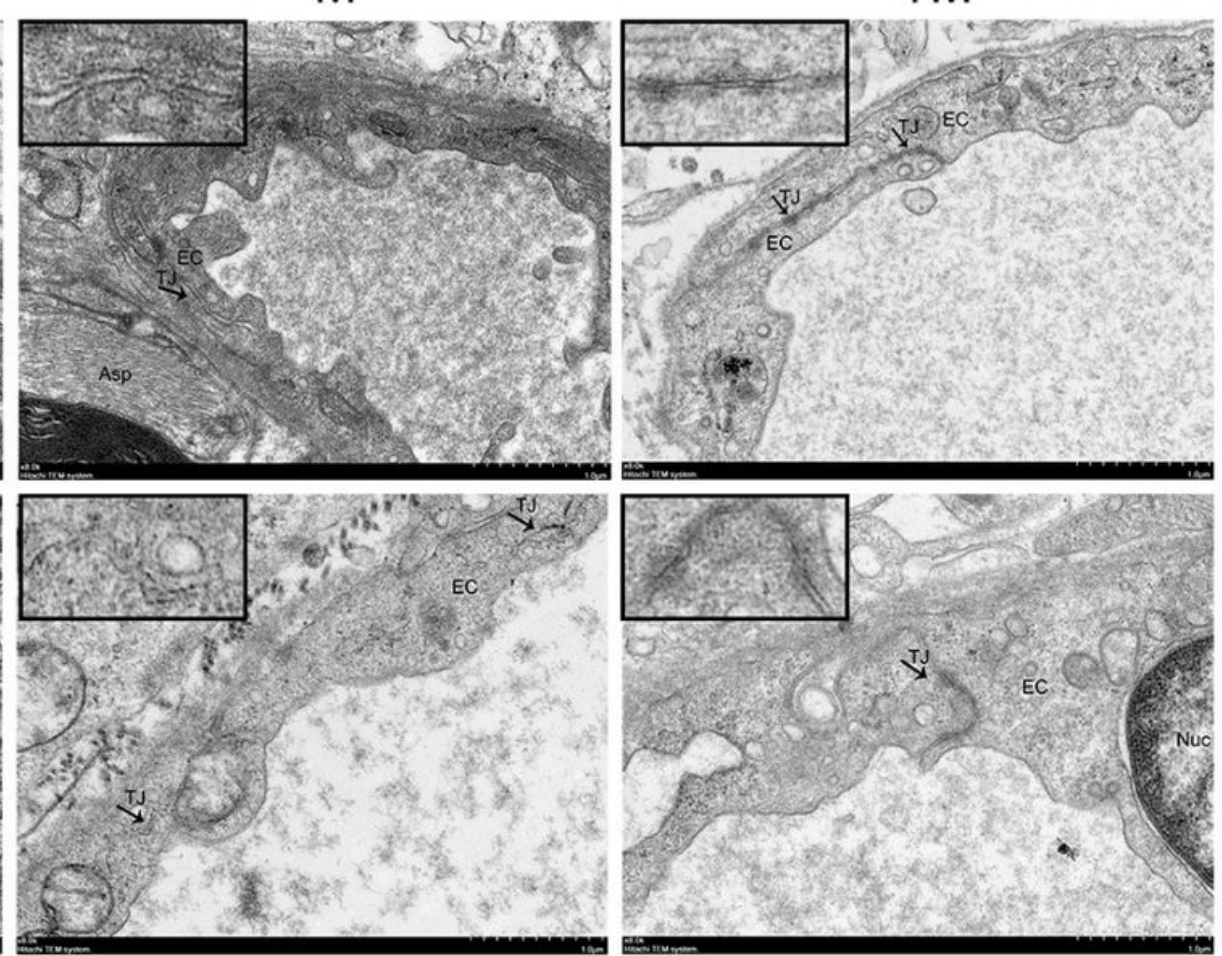

Figure 6

TT inhibits the expression of MMP-2 and MMP-9 after SCl. (a-c) Represent western blots and quantification data of MMP-2/9 in each group, columns represent mean $\pm S D, n=5$. \#p $<0.05$ as $M$ group versus $S$ group, ${ }^{*} p<0.05$ as TM group versus $M$ group. (d) TEM showed the EC-EC junctions in $S, M, T M$. Arrows indicate TJ electron dense band and the open TJ, scale bars are $1.0 \mu \mathrm{m}$. (\#p, ${ }^{\star} p<0.05 ; \# \# p, \star \star p<$ $\left.0.01 ; \# \# \# p,{ }^{\star * \star} p<0.001\right)$ 


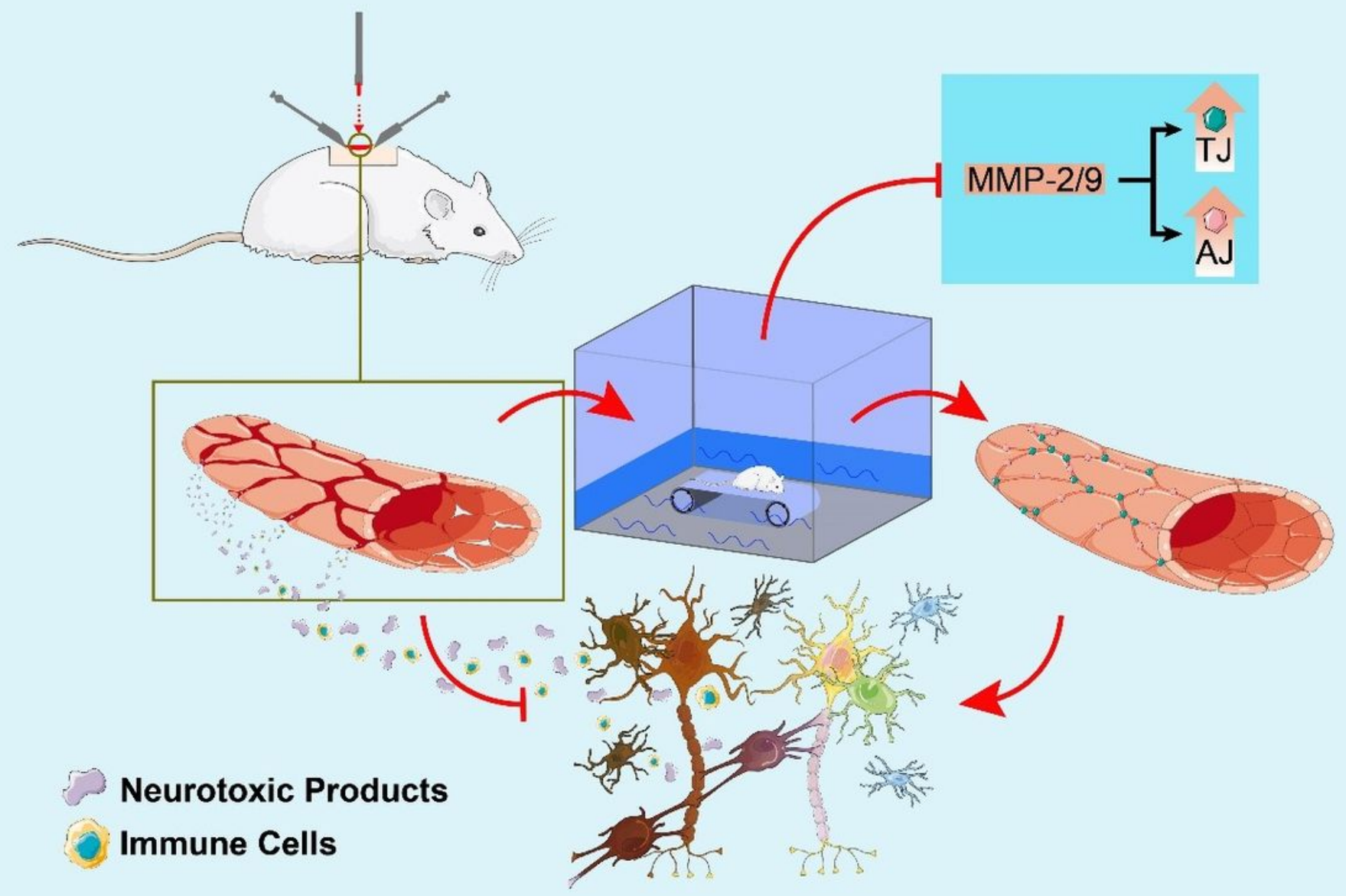

Figure 7

Proposed mechanism for the BSCB protection of water treadmill training after SCl. 